\title{
2008/82
}

How stochasticity and emergencies disrupt the surgical schedule

Jean-Sébastien Tancrez, Benoît Roland, Jean-Philippe Cordier and Fouad Riane 
CORE

Voie du Roman Pays 34

B-1348 Louvain-la-Neuve, Belgium.

Tel (32 10) 474304

Fax (32 10) 474301

E-mail: corestat-library@uclouvain.be http://www.uclouvain.be/en-44508.html 


\title{
CORE DISCUSSION PAPER
}

$2008 / 82$

\section{How stochasticity and emergencies disrupt the surgical schedule}

\author{
Jean-Sébastien TANCREZ1 ${ }^{1}$, Benoît ROLAND², \\ Jean-Philippe CORDIER ${ }^{3}$ and Fouad RIANE ${ }^{4}$
}

December 2008

\begin{abstract}
In health care system, the operating theatre is recognized as having an important role, notably in terms of generated income and cost. Its management, and in particular its scheduling, is thus a critical activity, and has been the sub ject of many studies. However, the stochasticity of the operating theatre environment is rarely considered while it has considerable effect on the actual working of a surgical unit. In practice, the planners keep a safety margin, let's say $15 \%$ of the capacity, in order to absorb the effect of unpredictable events. However, this safety margin is most often chosen sub jectively, from experience. In this paper, our goal is to rationalize this process. We want to give insights to managers in order to deal with the stochasticity of their environment, at a tactical-strategic decision level. For this, we propose an analytical approach that takes account of the stochastic operating times as well as the disruptions caused by emergency arrivals. From our model, various performance measures can be computed: the emergency disruption rate, the waiting time for an emergency, the distribution of the working time, the probability of overtime, the average overtime, etc. In particular, our tool is able to tell how many operations can be scheduled per day in order to keep the overtime limited.
\end{abstract}

Keywords: health care, surgical schedule, emergencies, Markov chain.

\footnotetext{
${ }^{1}$ Université catholique de Louvain, CORE, B-1348 Louvain-la-Neuve, Belgium; Catholic University of Mons, Louvain School of Management, B-7000 Mons, Belgium. E-mail: js.tancrez@uclouvain.be.

${ }^{2}$ Catholic University of Mons, Louvain School of Management, B-7000 Mons, Belgium.

E-mail: roland@fucam.ac.be

${ }^{3}$ Catholic University of Mons, Louvain School of Management, B-7000 Mons, Belgium.

E-mail: cordier@fucam.ac.be

${ }^{4}$ Catholic University of Mons, Louvain School of Management, B-7000 Mons, Belgium.

E-mail: riane@fucam.ac.be
}

This paper presents research results of the Belgian Program on Interuniversity Poles of Attraction initiated by the Belgian State, Prime Minister's Office, Science Policy Programming. The scientific responsibility is assumed by the authors. 



\section{Introduction}

In recent years, throughout Europe, the hospital environment has been subject to profound changes. Authorities introduced numerous reforms in order to rationalize health care expenses and to improve the citizens' quality of service. In addition to these structural challenges, health care management is made more and more complex and critical by various factors: the continuously growing demand for medical care, the ageing population, and the evolution of the pathologies, among others. This constrains hospitals to manage more efficiently their human and material resources, in order to reduce the costs while preserving the quality of care.

In this context, efficiently managing the operating theatre is a crucial question for hospitals. Indeed, the operating theatre plays a critical role in the hospital. It is an important activity for care creation, and it generates large income. However, it is also well known that the surgical units are one of the most important sources of expenses, with up to $10 \%$ of the hospital budget [2]. A more effective management of the operating rooms comes with a more rational use of the surgical resources, and consequently with a refined planning of the surgical units. The planning of the operating theatre aims at optimally scheduling the surgical operations that will take place during a period of time, often one or two weeks, on the basis of the demand coming from the surgical units or from the physicians. Each elective surgery is assigned to a given day, taking into account human and material constraints.

However, the actual realization of the planning cannot be perfectly predicted: the reality is stochastic. The length of a planned (or elective) operation may vary, its length cannot be predicted exactly. Moreover, the planning can be disrupted by some unplanned, urgent, operations, i.e. some emergencies. Emergencies may come from outside, but also from inside the hospital, if a patient's condition evolves unexpectedly. Obviously, emergencies cannot be planned as elective operations can, but may cause significant disturbance to the operating schedule. Furthermore, the length of an emergency operation is also stochastic. For the moment, in practice, most managers build schedules that fill the operating rooms for a given fraction of the available time (the occupation rate) and save a predetermined per-

The work of J.-S. Tancrez has been funded by the European Social Fund and the Walloon Region of Belgium. The work of J.-P. Cordier has been funded by Research Program IAP 6/09 "Higher Education and Research" of the Belgian Federal Authorities. Authors are also grateful to Professor P. Chevalier and to their colleague J.-C. Van den Schriek for many helpful discussions. 
centage of it (often about 15\% [17]). They keep a safety margin that will allow absorption of the stochasticity, the unpredictable events. They hope that the unallocated working time will be sufficient to deal with the unpredicted long operations, and to treat the emergencies. Doing this, their goal is to avoid getting too much overtime. In practice, this process is not rationalized. Most often, the occupation rate is set subjectively, according to people's experience or by benchmarking.

This paper aims to bring some insights to take account of the stochastic reality, in order to better manage the operating theatre, at a tacticalstrategic decision level. Our goal is to propose tools to operating theatre managers in order to help them deal with the randomness of their environment, coming from the random operating times as well as from the unpredictable arrival of emergencies. In particular, our work should provide measures allowing them to rationally choose the occupation rate to be applied in their operating rooms. From the configuration of the operating theatre and the arrival rate of the emergencies, our tool is able to compute the occupation rate of the operating theatre which will lead to a chosen, acceptable, probability of overtime. To achieve this, we propose an analytical model of the operating theatre, based on a Markov process.

In this work, we thus choose to analytically treat the operating theatre. Mathematical and simulation approaches have their own advantages and can be considered as complementary: they are often used to validate each other. However, in the present case, the mathematical approach can be justified and shown to be appropriate. Our work is positioned at the tactical-strategic level of decision. From few data on the system, we want to propose measures on the behavior of the latter and how to better manage it. In this context, the main advantage of the simulation, i.e. its ability to model in more details, is not justified, or fruitful, since simulation needs a deeper knowledge of the system than we consider. For the problem we are concerned with, the analytical approach has the advantage of quickly offering reliable and constant performance measures, requiring few data.

A quite vast literature exists on patient flow management, see [8]. About the scheduling of the operating theatre in particular, we refer the interested reader to Cardoen et al. [1] which gives a substantive state of the art. In the following, we briefly sketch the literature related to stochastic modelling in health care management. It is structured according to the tools applied: queueing theory, Markov theory and simulation.

First, queueing theory has been applied in various studies, in health care management. Green [7], Preater [19] provide good reviews. The majority of these studies are concerned with the sizing of critical resources (the beds, 
for example, [10]) or with the allocation of resources [6]. For this, Kim et al. [11] focus on the evaluation of the waiting time to enter an intensive care unit. Concerning the emergency flow, de Bruin et al. [3] applied queueing theory to model the cardiac in-patient flow, and studied the occurrence of bottleneck.

Second, Markov chains have been shown to be useful in patient flow modelling. For the sizing of critical resources, Harrison et al. [9] showed that they offer a more detailed modelling, compared to queueing theory, and allow to compute the overflow and to take account of the variability. McClean et al. [16] applied Markov theory and used phase-type distributions to compute the length of stay in a geriatric care unit. The use of phase-type in health care management has been reviewed in [4].

Third, the simulation approach has been extensively used to deal with particular cases, taking advantage of its main asset: its ability to model specific systems in details. In particular, the emergency patient flow, which is inherently stochastic, has been studied by various authors. Kolker [12] studies the patient flow to an emergency care unit. Komashie and Mousavi [13] try to understand the behavior of the system with regard to the hidden causes of excessive waiting times. Ruohonen et al. [20] explore the possibility of a triage-team in order to better allocate the resources.

In our work, we consider the stochasticity brought into the operating theater schedule by two different patient flows: elective operations and emergencies. In the literature, emergencies have been studied moderately. Stochastic operating times of elective operations are sometimes considered in the planning literature, but most often by simulation (see also [15]). However, to the extent of our knowledge, very few papers consider both patient flows and incorporate the emergencies in the operating theatre planning problem. Gerchak et al. [5] propose a stochastic dynamic programming model for the aggregate advance scheduling problem. They aim at determining how many of the additional requests for elective surgery can be assigned for each day, according to emergencies and already scheduled operations. They maximize an expected profit function while penalizing overtime and postponements. They consider the total daily working time used by emergencies as well as elective operating times as random variables. They propose no other performance measures than those connected with their profit function. A second example is given by [14], where the authors propose a method to build an operating programme, taking account of emergencies. However, they consider emergencies through a stochastic time needed in the planning, avoiding measures such as waiting time, and the elective operating time is deterministic. None of these approaches consider the possibility to dedicate rooms 
to emergencies. Moreover, we found no research that clearly justifies how to set the minimum room occupation rate in order to absorb unpredictable events.

The rest of the paper is organized as follows. Section 2 describes our modelling of the operating theatre, taking its stochasticity into account. Section 3 details the Markov process used to analytically model the system and to compute performance measures. Section 4 illustrates the utilization of the proposed tool, by some experiments and examples. Concluding remarks are given in Section 5 .

\section{Modelling of the operating theatre}

As previously said, our goal is to help managers to deal with the randomness of their environment, to help them planning the operations by taking the unpredictable events into account. The stochasticity essentially comes from two facts. First, the length of the operations cannot be exactly predicted. Second, emergencies arrive unexpectedly, and cause a significant disruption to the surgical schedule.

In our model, we thus consider stochastic operating times and we differentiate scheduled operations and emergencies. In particular, we want to measure the disruption of the operating schedule by the emergencies. Indeed, when the emergency flow is sizeable, emergencies may significantly disrupt the schedule. This affects the quality of service to elective operations as well as to emergencies. In this context, an option could be to separate the flows, by dedicating one or more operating rooms to emergencies. This would improve the care provided to the emergencies, by reducing their waiting time, as well as to the planned operations by avoiding too much disruption and delay. However, dedicating an operating room lowers the available time for elective operations, as some rooms are not accessible to them anymore. We thus included this option in our model: we differentiate dedicated (to emergencies) rooms and versatile rooms (which treat scheduled operations and, if necessary, emergencies). We suppose that the versatile operating rooms can accommodate any kind of surgery treated in the hospital [14]. In the following, an operating theater with $n_{v}$ versatile rooms and $n_{d}$ dedicated rooms will be said to have a $\left[n_{v} \mid n_{d}\right]$ configuration.

This leads us to the model illustrated in Fig. 1. In this model, the scheduled operations are treated in the versatile rooms. We thus consider that the versatile operating rooms are continuously fed by a flow of elective operations. This arrival process is said to be saturated. In other words, as 


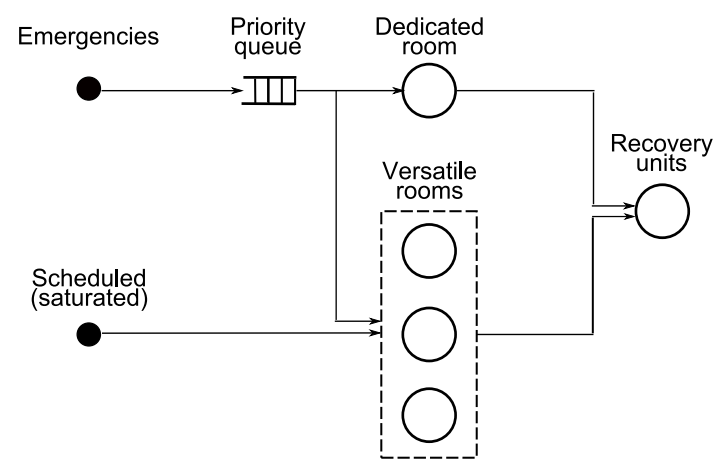

Figure 1: Operating theatre made of four rooms, of which one is dedicated to the emergencies and three are versatile ([3|1] configuration).

these operations are planned, the next elective surgery is always ready to start when an operation ends. On the contrary, emergencies arrive following a random process. We consider emergencies as events to carry out as a priority. They represent critical cases that have to be treated in the day. When an emergency arrives, it goes to a priority queue (of infinite capacity), i.e. an emergency has priority over an elective case. In concrete terms, when an emergency arrives, it goes directly to the dedicated rooms (if the configuration of the surgical units contains some), if one is vacant. If not, it waits in the queue till one operation ends in any room (dedicated or versatile). If the first operation to end is in a versatile room, the emergency has priority over the planned operation, and delays the latter. This is one of the main sources of disruption of the planning (another source of disruption are the stochastic operating times). Once an operation is performed (in a stochastic time), the patient is taken to the recovery units. Although, recovery beds management is a proper problem, and is not addressed in this work. Finally, note that the model can be studied without any dedicated room, i.e. every emergency treated in the versatile rooms.

We now list the modelling assumptions we make. They allow us to analytically model the operating theatre depicted in Fig. 1 by a Markov process (see the next section). However, note that we argue in Sect. 3.2 that these assumptions are not restrictive. We validate them by comparing the results of the analytical modelling with simulation results. We essentially make three assumptions:

- The operating time of a planned operation is supposed to be exponentially distributed with rate $\lambda_{p}$, expressed in operations/day, where a 
working day is supposed to count 8 hours. We decide not to distinguish the elective operations. We could differentiate the surgeries by room, but it would lead to a much larger Markov chain. Furthermore, we can consider that the difference in the operations is modelled by their stochastic nature.

- We suppose that the emergencies arrive according to a Poisson process, with arrival rate $\lambda_{i}$. This is a classical assumption in the literature. It has been validated in many contexts (see [7] for example).

- We also assume that the operating time of an emergency is exponentially distributed, with rate $\lambda_{e}$. Note that the elective operations and emergencies processing times may include the preparation (preoperative) and the cleaning (postoperative) of the room.

The modelling of the operating theatre is now defined. Our main objective is to evaluate the effect of stochasticity on the surgical schedule, with various performance measures. From the managerial viewpoint, the tool should allow us to compute measures such as the arrival rate of the emergencies in the planning, the operating time of these emergencies, the probability of overtime, and the desirable occupation rate. Concerning the quality of care for emergencies, measures of interest are, for example, the average waiting time for an emergency or the probability to wait more than one hour. Furthermore, we would like to evaluate the opportunity to add or remove dedicated (to emergencies) operating rooms.

\section{The Markovian model}

In this section, we present an analytical model of the operating theatre described in the previous section. The assumptions required by the analytical model are next validated by simulation. The problem in hand clearly brings queueing theory to mind (see Fig. 1). However, the organization of the system, in particular the differentiation of the dedicated rooms and the versatile rooms, prevent the application of this theory. For example, the arrival process of the emergencies to the versatile rooms cannot be considered as Poisson. The emergencies reach the versatile rooms only when the dedicated room(s) is (are) busy, i.e. intermittently. The complexity of the system leads us to model the evolution of the operating theatre by a Markov process, as described below. 


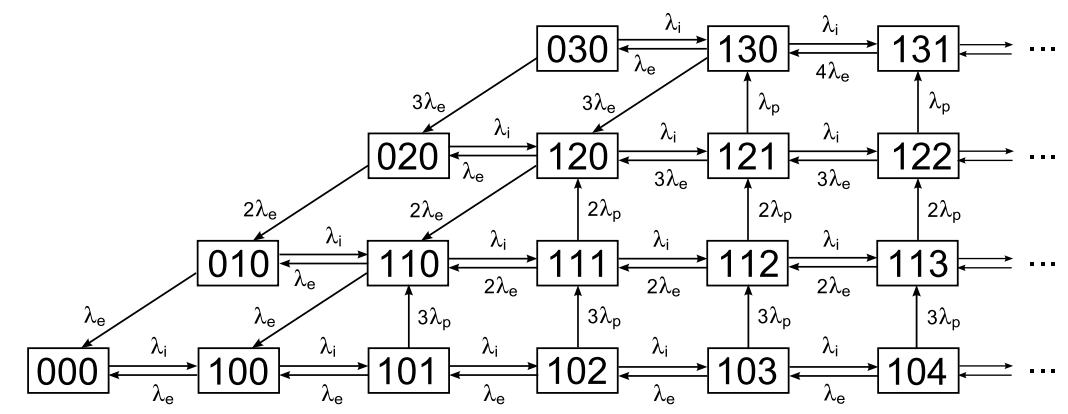

Figure 2: Continuous Markov chain for an operating theatre made of four rooms, of which one is dedicated to the emergencies and three are versatile ([3|1] configuration).

\subsection{The Markov process}

As detailed in Sect. 2, we suppose the operating times to be exponentially distributed (with rates $\lambda_{p}$ and $\lambda_{e}$ ) and the emergency arrival process to be Poisson (with rate $\lambda_{i}$ ). These assumptions allow us to model the operating theatre as a continuous Markov process.

The main goal of this Markov process is to measure the disturbance brought by the emergencies in the schedule. We thus focus on them to build the Markov chain (see the example given in Fig. 2). Each state $d v q$ of the chain is defined by the number of emergencies in each part of the system. Each number in the state $d v q$ refers to one part of the operating theatre: the dedicated rooms $(\underline{d} v q)$, the versatile rooms $(d \underline{v} q)$, and the emergencies priority queue $(d v q)$. For example, the state $d v q=112$ represents an operating theatre where one emergency is under treatment in the dedicated room, one emergency is inserted in the versatile rooms, and two emergencies are waiting for a room to become available.

The transitions between the states correspond to the different events that may occur in a given state. These events are of four types:

- When an emergency arrives (with rate $\lambda_{i}$ ), it enters in a dedicated room if possible (transition 020 to 120 for example), otherwise it joins the queue (112 to 113).

- When an emergency ends in a dedicated room (with rate $n_{e d} \lambda_{e}, n_{e d}$ being the number of emergencies in progress in the dedicated rooms), it leaves the corresponding room. If another emergency is waiting for 
treatment, it replaces the finished one in the same operating room (112 to 111). Otherwise, the queue stays empty and the dedicated room becomes free (120 to 020).

- When an emergency leaves a versatile room (with rate $n_{e v} \lambda_{e}, n_{e v}$ being the number of emergencies in progress in the versatile rooms), it is replaced by another emergency if one was waiting (112 to 111, again). If the queue was empty, a scheduled operation may enter the room (120 to 110$)$.

- The last case corresponds to an elective operation ending (with rate $n_{p} \lambda_{p}, n_{p}$ being the number of elective operations in progress, i.e. the number of versatile rooms minus $n_{e v}$ ). If an emergency is waiting for treatment, the emergency reaches the versatile room (112 to 121). If not, a new planned operation enters the room, and the state stays the same (120 for example).

As the states and transitions are defined, the continuous Markov chain modelling the system can be built. It is illustrated in Fig. 2 for an operating theatre composed of four operating rooms with a [3|1] configuration, i.e. one is dedicated to emergencies. It can be seen that the structure of the chain is different depending on the presence of emergencies in the queue or not, as can be deduced from the description of the various transitions. We implemented this Markov process, to get a tool as flexible as possible. The different rates $\left(\lambda_{i}, \lambda_{e}\right.$ and $\left.\lambda_{p}\right)$, the number of operating rooms, as well as their repartition in dedicated and versatile rooms, can be chosen. Moreover, the Markov process offers a complete modelling of the evolution of the emergencies in the operating theatre. Consequently, various performance measures can be derived from it. We describe the most relevant ones in the following.

First, the implemented tool computes the stationary probabilities of the states of the Markov chain. Informally speaking, the stationary probability of one state gives the proportion of time during which the operating theatre is in this state. In order to compute them, we fix a maximum size for the priority waiting queue, so that the Markov chain becomes finite. This size is chosen so that the ignored state probabilities are negligible, i.e. so that the final results are not affected. The stationary probabilities $\pi_{i}$ of the states of a continuous finite Markov process can be easily computed with the classical formula $\pi Q=0$, with $\sum_{i} \pi_{i}=1$ and where $Q$ is the transition matrix of the Markov process. From the stationary probabilities, we can derive several measures (with any parameter choice): 
- The disruption rate, denoted $\lambda_{i p}$, is the average rate of the emergencies entering the versatile rooms. In other words, it reflects the disturbance of the schedule by the emergencies. It is computed as the sum of the rates of the transitions leading to such a disruption, weighted by the stationary probabilities of the corresponding states. Note that if no operating room is dedicated to emergencies, we have $\lambda_{i p}=\lambda_{i}$.

- The disruption rate can be straightforwardly translated into the working time needed to treat the emergencies entering the versatile rooms. The latter gives a first idea of the time slot that the planner should reserve for emergencies, on average.

- In order to evaluate the quality of treatment of the emergencies, we compute the probability for an emergency to wait (it equals one if no room is dedicated to the emergencies). It equals the probability for an emergency to arrive when all the dedicated rooms are busy, i.e. the sum of the stationary probabilities of the states showing this characteristic.

- Again, with the same goal, we can evaluate the average waiting time in the queue for an emergency. It can be computed using the Little's law: the arrival rate is deduced from the waiting probability (see previous item) and the average number of emergencies in the queue is easily inferred from the stationary probabilities.

- The distribution of the waiting time can also be inferred from the Markov chain and its stationary probabilities. It can be shown that this distribution is continuous phase-type [18]. From the distribution, the probability for an emergency to wait more than a given time, one hour for example, can be found.

- Many other measures could be computed such as: the average number of emergencies in the operating theatre, the average occupancy rate of the dedicated rooms, the average number of emergencies in the queue, etc.

However, even if these measures give interesting insights, the stationary probabilities do not allow to compute the overtime. At this stage, we are not able to answer our main question: which occupancy rate should be taken by the planner in order to reach a chosen maximum overtime ? In order to compute the overtime, we need to find the distribution of the global operating time for a day, for planned operations as well as for emergencies. 
For this, we first need to find the distribution of the number of emergencies disrupting the planning in one day, i.e. the probabilities to have zero, one, two, etc. emergencies operated in the versatile rooms.

In order to compute the distribution of the number of disrupting emergencies, we extend the Markov chain. A fourth attribute (dvqp) is added to each state of the chain. It counts the number of emergencies which entered the versatile rooms. The transitions are thus modified accordingly: each transition which corresponds to an emergency entering the versatile rooms now goes from $d v q \underline{p}$ to $d^{\prime} v^{\prime} q^{\prime} p+1$ (where $d^{\prime} v^{\prime} q^{\prime}$ stays the same as for the previous Markov chain). For example, for an operating theatre with a [3|1] configuration (three versatile rooms and one dedicated room), from state 1121, if the emergency currently operated in a versatile room ends, a waiting emergency will enter the corresponding room, leading to state $111 \underline{2}$ (with rate $\lambda_{e}$ ). If one elective operation ends, it will lead to state $121 \underline{2}$ (with rate $2 \lambda_{p}$, similarly to the chain given in Fig. 2), one waiting emergency entering the versatile room. The Markov chain is thus very similar to the one presented in Fig. 2. Informally said, the new Markov chain has one more dimensionality, and transitions occurs from one level to the upper level ( $d v q p$ to $\left.d^{\prime} v^{\prime} q^{\prime} p+1\right)$ if an emergency enters the versatile rooms.

From the extended Markov chain, the transient probabilities $\pi(t)$ of the states can be computed, using the classical equation $\pi(t)=\pi(0) e^{Q t}$ (see [21] for example), where $Q$ is the transition matrix and $\pi(0)$ gives the initial condition $^{1}$. The transient probabilities $\pi(8$ hours $)$ give the probability of each state at the end of a working day. By construction of the extended chain, we thus get the probability that, for example, two emergencies disrupt the planning during one day. For this, we just need to sum the transient probabilities of every state $d v q 2$ of the extended Markov chain. Finally, we can thus compute the distribution of the number of emergencies disrupting the schedule. Note that, again, we truncate the chain in order to make it finite, removing states with negligible probabilities.

From the distribution of the number of disrupting emergencies, we can infer the distribution of the global working time in the versatile rooms. The latter distribution appears to be a continuous phase-type distribution. A phase-type distribution is a complex composition of exponential distributions which can be modelled as the time until absorption of a Markov process with one absorbing state. It is fully characterized by the transition matrix of the Markov process and the initial probabilities of starting in any of the

\footnotetext{
${ }^{1}$ Here, we suppose that, at the beginning of the day, there is no emergency in the operating theatre, i.e. $\pi_{0000}(0)=1$.
} 


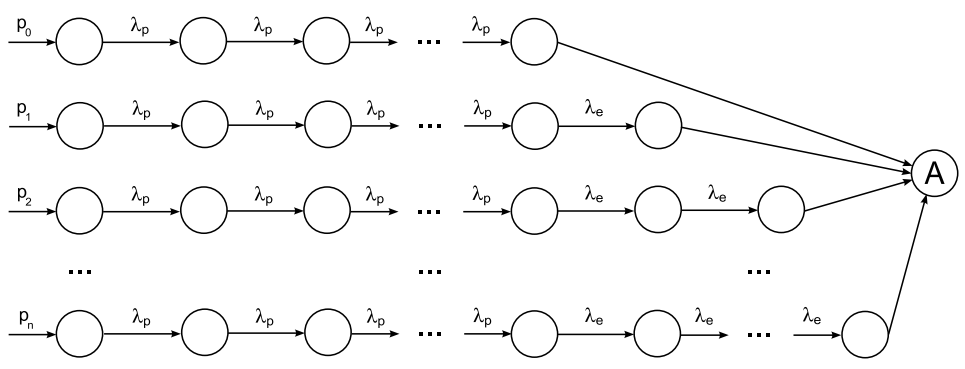

Figure 3: Continuous phase-type distribution of the global working time in the versatile operating rooms, for one day.

states of the chain [see 18]. To begin, suppose there are, on a particular day, two emergencies which disrupt the surgical schedule. In this case, the global working time in the versatile rooms will be composed of $N_{p}$ planned operations and two urgent operations. To get the total working time on all versatile rooms, we just have to sum all the exponentially distributed times of these operations (planned and urgent). In terms of Markov process, it can be modelled by $N_{p}+2$ states in series (with transition rates $\lambda_{p}$ for the planned operations and $\lambda_{e}$ for the emergencies, see the third "branch", from top, of the chain in Fig. 3). However, the number of emergencies disrupting the planning is not fixed: it is random. The number of emergencies to be added to the $N_{p}$ planned operations changes accordingly (see the "branches" for $0,1,2$ and $n$ emergencies in Fig. 3). As the distribution of the number of disruptions can be computed (see previous paragraph), the probability of each case is known. It leads us to the phase-type distribution illustrated in Fig. 3. The first "branch" of the Markov process corresponds to a day without disruption, the second branch to a day with a single disruption, etc. The initial probabilities to enter each branch $\left(p_{0}, p_{1}\right.$, etc.) equal the probability to have zero, one, etc. disruptions on a given day. The time to reach the end of a "branch", i.e. the absorbing state $A$, equals the sum of the operating times, with the right number of operations of each type and the right transition rates.

Fortunately, the theory on phase-type distribution is well established [see 18]. The density function and the distribution function, as well as the moments (like the mean and the variance), are known, from the transition matrix and the initial probabilities. We are able to compute them. It allows us to compute the overtime. Indeed, the probability of overtime corresponds to the probability that the working time in the versatile rooms exceeds 8 hours per room, i.e. $1-F\left(n_{v} 8\right.$ hour $\left.s\right)$ where $F(t)$ is the cumulative 
distribution function of the distribution of the global working time, which is known, and $n_{v}$ the number of versatile rooms. We built the tool we were looking for: we are able to link the occupancy rate planned by a manager and the probability to have overtime. It allows to answer questions such as: what occupancy rate should be chosen, in other words how "loaded" should be the operating programme, to have no more than one day of overtime per week, on average?

\subsection{Validation by simulation}

The Markovian process detailed in the previous section enables us to analytically model the operating theatre described in Sect. 2. Nevertheless, it requires some technical assumptions on the statistical distributions of the events. Modelling the unpredictable arrival of emergencies as a Poisson process is a classical assumption. However, the validity of the assumptions is not as obvious on the processing times of the various operations. In this section, we therefore want to check the robustness of our theoretical results according to the variability of the statistical distributions of the operating times.

We used the software AnyLogic [22] to develop a simulation model that depicts the problem we are concerned with. Our goal is to validate the assumptions on the statistical distributions of the operating times. For that purpose, we assess how the disruption rate $\lambda_{i p}$ evolves according to the variability of these distributions. As shown later, we can argue that our Markovian model is accurate and that the required assumptions alter very slightly the final results.

Using simulation, we estimate the performances of an operating theatre composed of seven rooms with a [6|1] configuration, i.e. six versatile rooms and one dedicated room. This configuration is the one used in a Belgium hospital we have contact with. The default values for the rates are $\lambda_{i}=4$ (four emergencies arrive a day, on average), $\lambda_{e}=4$ (four emergencies are treated a day, by room, on average) and $\lambda_{p}=3$ (three elective cases are treated a day, by room, on average). We compare in Table 1 the analytical and simulation values of the disruption rate $\lambda_{i p}$, i.e. the arrival rate of emergencies in the versatile rooms. The arrival process of emergencies in the operating theatre is Poisson with rate $\lambda_{i}$ ranging from 2 to 5 . The simulated operating times of emergencies follow a Gamma distribution with a rate of 4 operations/day, while the simulated operating times of the elective cases follow a Gamma distribution with rate of 3 operations/day. The parameters of the Gamma distributions are chosen so that the coefficients of variability 
Table 1: Analytical and simulated values of the arrival rate $\lambda_{i p}$ of emergencies in the versatile rooms ([6|1] configuration). The simulated operating times follow Gamma distributions with various coefficients of variability $c v$.

\begin{tabular}{ccccccc}
\hline Arr. r. & Anal. & $c v=0.5$ & $c v=0.75$ & $c v=1$ & $c v=1.25$ & $c v=1.5$ \\
\hline$\lambda_{i}=2$ & 0.58 & $0.58(.01)$ & $0.58(.01)$ & $0.59(.01)$ & $0.58(.01)$ & $0.58(.01)$ \\
$\lambda_{i}=3$ & 1.14 & $1.15(.01)$ & $1.16(.02)$ & $1.15(.01)$ & $1.15(.01)$ & $1.16(.02)$ \\
$\lambda_{i}=4$ & 1.81 & $1.81(.01)$ & $1.81(.02)$ & $1.82(.02)$ & $1.82(.01)$ & $1.82(.02)$ \\
$\lambda_{i}=5$ & 2.54 & $2.55(.02)$ & $2.55(.02)$ & $2.57(.02)$ & $2.55(.02)$ & $2.55(.02)$ \\
\hline
\end{tabular}

$c v$ range from 0.5 to 1.5 . The simulation time equals 10,000 days, and each simulation is replicated 10 times. Table 1 shows the average $\lambda_{i p}$ and, between brackets, the corresponding standard deviations.

On Table 1, it obviously appears that the disruption rates $\lambda_{i p}$ are highly homogeneous regarding the statistical distribution variability $(c v)$. The difference between Markovian and simulation results are negligible. The Markovian approach is proved to be robust to the variability.

\section{Computational results}

In the previous sections, we developed a model of the operating theatre, paying particular attention to its stochasticity. From it, we built a flexible tool which computes various performances measures. The goal is to help operating theatre managers to take the stochasticity of their environment into account, especially when planning the operations. Moreover, we pay special attention to the disruption of the schedule caused by the emergencies. In the present section, we illustrate how our tool can be used, and how it provides insights about the behavior of the operating theatre. We also show how the various parameters (rates and numbers of rooms) affect the performance measures. By default, we will suppose the operating theater to show a [6|1] configuration such as in the Belgium hospital we have contact with $^{2}$. The default values for the rates are $\lambda_{i}=4$ (four emergencies arrive a day, on average), $\lambda_{e}=4$ (four emergencies are treated a day, by room, on average) and $\lambda_{p}=3$ (three elective cases are treated a day, by room, on average).

\footnotetext{
${ }^{2}$ In this hospital, the average operating time approximately equals three hours for an elective operation and two hours for an emergency.
} 


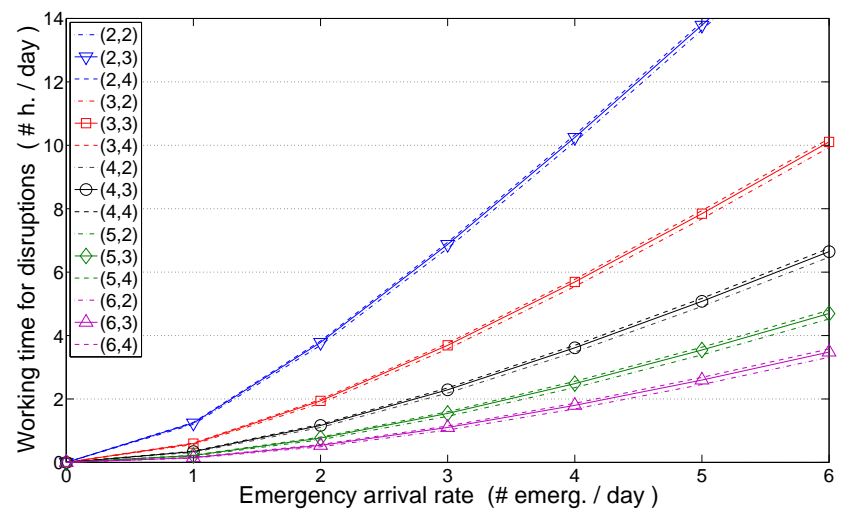

Figure 4: Operating time of the disrupting emergencies, with rates $\lambda_{i}, \lambda_{e}$ and $\lambda_{p}$ varying, for a [6|1] configuration. The legend shows the couple $\left(\lambda_{e}, \lambda_{p}\right)$.

First, we analyse how the rates of the operating time distributions and of the Poisson emergency arrival affect the disruption of the surgical schedule. Figure 4 shows how the working time needed to treat the disrupting emergencies changes when the rates $\lambda_{i}, \lambda_{e}$ and $\lambda_{p}$ vary. For example, with the default rates (i.e. four emergency arrive per day while four can be treated per day, which means that, in a deterministic environment, every emergency could be treated in the dedicated room), approximately four hours of operation, on average, will be needed in the versatile rooms to operate the emergencies. In other words, in this case, about two (1.81) emergencies among four will enter the versatile rooms and disrupt the elective operations. This clearly reflects the importance of considering the stochasticity of the hospital environment. It can also be seen on Fig. 4 that, unsurprisingly, when the arrival rate of the emergencies increases, the disruption of the schedule increases. Similarly, when the time to operate emergencies increases $\left(\lambda_{e}\right.$ decreases), their impact is bigger. Concerning the rate $\lambda_{p}$ to operate elective cases, Fig. 4 shows that it has little influence. This observation tends to validate our assumption about the non-differentiation of the elective operations (see Sect. 2): specifying the lengths of the elective operations would not have much impact on the results, for our problem.

It is also of interest to see how the operating theatre configuration influences the disruption of the planning. Figure 5 shows how the emergency arrival rate into the versatile rooms decreases when the number of dedicated 


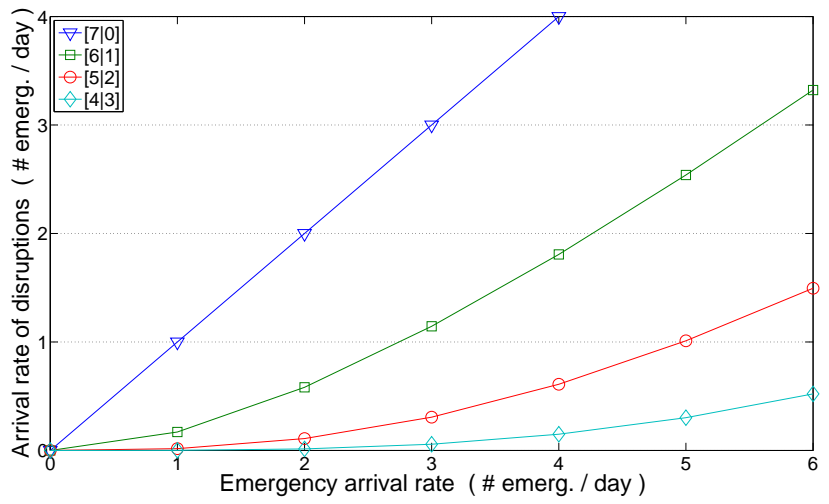

Figure 5: Arrival rate of the disrupting emergencies, with $\lambda_{i}$ increasing $\left(\lambda_{e}=4\right.$ and $\left.\lambda_{p}=3\right)$, and for various operating theatre configurations $\left[n_{v} \mid n_{d}\right]$.

rooms increases. With the default rates $\left(\lambda_{i}=4, \lambda_{e}=4\right.$ and $\left.\lambda_{p}=3\right)$, if we dedicate two rooms to emergencies instead of one, the arrival rate of the disrupting emergencies decreases from 1.81 to 0.61 .

These performance measures are related to the disruption of the schedule by the emergencies. A second kind of measures reveals the quality of service to the emergencies, which is mainly connected to their waiting time. Figure 6 shows how the average waiting time, for an emergency, is affected when the rates $\lambda_{i}, \lambda_{e}$ and $\lambda_{p}$ vary. For the default example, an emergency will have to wait 14 minutes on average, which looks reasonable. Note that, without dedicated room, it would have to wait 26 minutes on average. It can be seen in Fig. 6 that the waiting time increases with the arrival rate of emergencies and with the average operating time of an emergency (as more emergencies have to wait). Moreover, the operating time of an elective operation has more impact than previously. This comes from the fact that a queueing emergency has to wait longer for an operation to end.

More detailed information about the waiting time comes with its distribution. In particular, it is relevant to check the probability for an emergency to wait what is considered as a too long time. For example, Fig. 7 shows the probability to wait more than one hour, for various operating theatre configurations. Again for the default example, the probability to wait more than one hour equals $5.3 \%$ (17.1\% for half an hour). A manager could consider this proportion as excessive. One option could be to dedicate another operating room to emergencies. In this case, the probability to wait more than one hour decreases to $2 \%$ (6.9\% for half an hour). Also note 


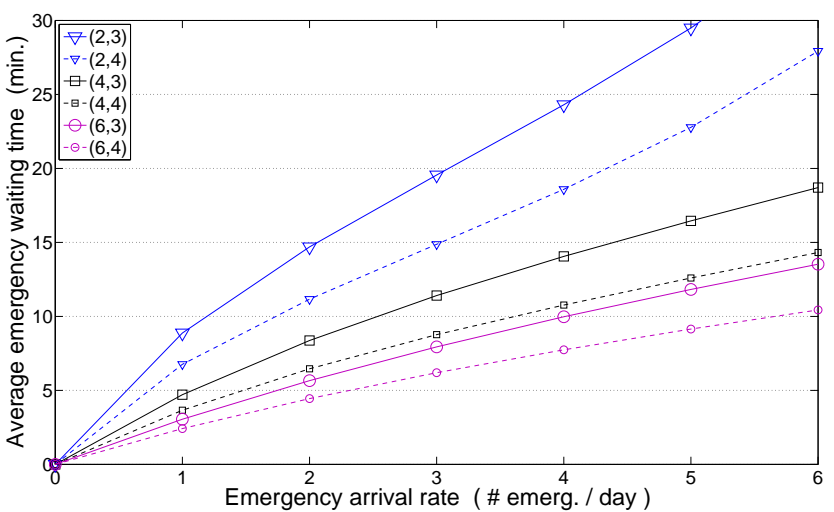

Figure 6: Average waiting time for an emergency, with rates $\lambda_{i}, \lambda_{e}$ and $\lambda_{p}$ varying, for a [6|1] configuration. The legend shows the couple $\left(\lambda_{e}, \lambda_{p}\right)$.

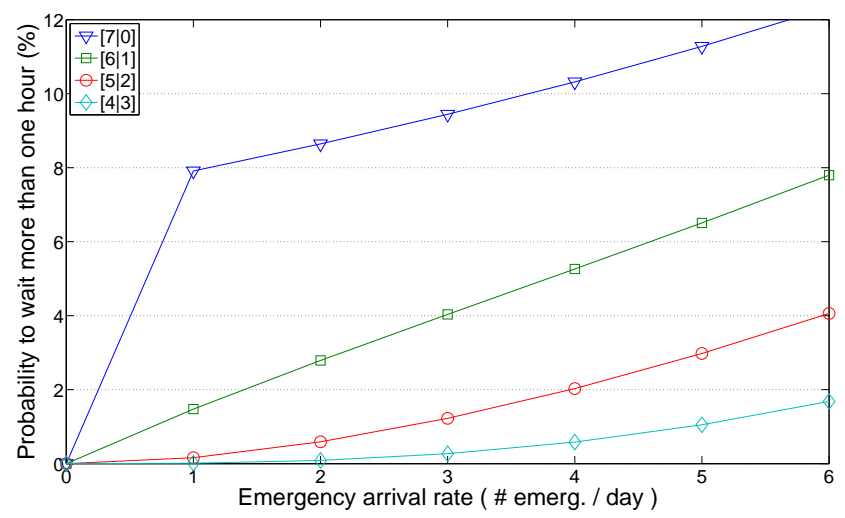

Figure 7: Probability to wait more than one hour, with $\lambda_{i}$ increasing $\left(\lambda_{e}=4\right.$ and $\lambda_{p}=3$ ), and for various operating theatre configurations $\left[n_{v} \mid n_{d}\right]$. 
that, without dedicated room, the probability would be $10.3 \%$ (32.3\% for half an hour), what could be considered as an argument for dedicating one room. Unsurprisingly, the waiting probability increases with the arrival rate of emergencies, and with the number of versatile rooms (for a given number of room).

We now analyse the overtime, which is one of the main concern of managers, as it is an important source of cost. By overtime, we mean here a working time exceeding the available time on all the versatile room $\left(n_{v} \cdot 8\right.$ hours $)$. Figure 8 reveals the probability of overtime according to the number of elective operations planned in the versatile rooms. This picture offers an answer to the following important practical question: what should the occupancy rate of the planning be in order to avoid getting more than, say, one day of overtime per week (a week contains here five working days)? For the default configuration $\left(\lambda_{i}=4, \lambda_{e}=4\right.$ and $\lambda_{p}=3$, configuration [6|1]), it can be seen that 13 operations can be scheduled in order to have a probability of overtime smaller than 0.2 , i.e. one day of overtime per week (of five days). If the manager decides to allow two days of overtime per week, he could then plan 16 operations per day. Another way to present these measures is to use the average occupancy rate: 13 planned operations per day is equivalent to an occupancy rate of $72.2 \%$ and 16 operations is equivalent to an occupancy rate of $88.9 \%$. However, this does not include the disrupting emergencies. Adding the emergencies, the versatile rooms will be busy $78.3 \%$ of the time, on average, if one day of overtime per week is allowed. It two days are considered as reasonable, the versatile rooms will be occupied $94.9 \%$ of the time, on average. It can also be seen on Fig. 8 that, when more emergencies are arriving in the hospital, less operations can be planned in order to keep the probability of overtime under the fixed treshold. When the number of versatile rooms decreases, the number of planned operations has to decrease, of course.

If the overtime is the main concern of a manager, he can also get a more detailed information thanks to the distribution of the working time. Figure 9 depicts this distribution for various allowed probability of overtime (one day every two weeks, one day a week, or two days a week). The probability that the working time exceeds 48 hours (6 rooms times 8 hours) equals the probability of overtime. The distribution provides various other informations. For example, it tells the manager that, in the case of two "overtime days" a week, there is $40.5 \%$ chance that the operations of the day will take less than 42 hours (i.e. 7 hours per room, on average). In other words, the manager now knows that two days a week, on average, the rooms, and the associated ressources, will be free for more than one hour. 


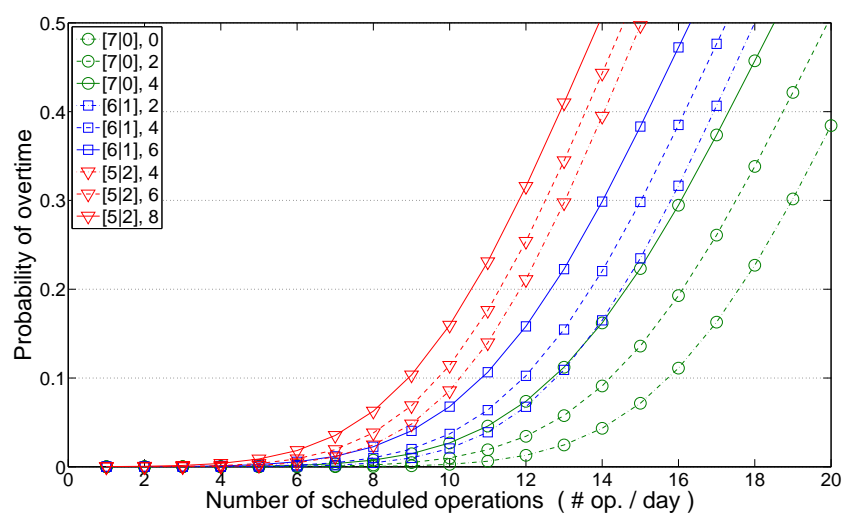

Figure 8: Probability of overtime, in function of the total number of scheduled operations per day. The legend shows the configuration $\left[n_{v} \mid n_{d}\right]$ and the rate $\lambda_{i}\left(\lambda_{e}=4\right.$ and $\left.\lambda_{p}=3\right)$.

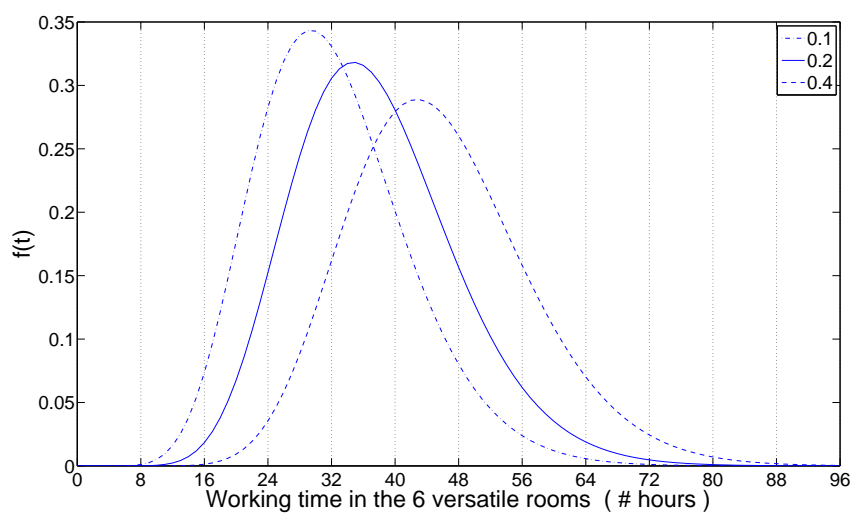

Figure 9: Probability density function of the working time in the versatile rooms ([6|1] configuration, $\lambda_{i}=4, \lambda_{e}=4$ and $\left.\lambda_{p}=3\right)$. The legend shows the chosen probability of overtime. 
The average amount of overtime can also be computed (and could have been chosen as the criteria to choose the occupancy rate). For example, in the case of two "overtime days" a week, the average overtime per room, if there is overtime, equals 1.49 hours. In other words, in this configuration, if the planner choose to plan sixteen operations per day, there will be overtime twice a week and for one hour and an half on average.

Finally, let us summarize the measures computed by our tool. We suppose a manager who would like to have insights about how to take account of the randomness when he plans the operations for the next week. The operating theater is composed of seven rooms. He estimates the number of emergencies per day to equal four, the operating times for elective and emergency operations to be, on average, 2 hours and 40 minutes, and 2 hours, respectively. The manager notices that the number of emergencies arriving corresponds to the number of emergencies that can be operated on per day. He thus thinks about dedicating one room to the emergency. The main advantage of this option is to reduce the waiting time of the emergencies, which is critical. He measures that, when the number of dedicated rooms goes from zero to one, the probability to wait more than half an hour goes from $32 \%$ to $17 \%$, while the probability for one hour goes from $10 \%$ to $5 \%$ (with two dedicated rooms, these probabilities drop off to $7 \%$ and $2 \%$, respectively). For the quality of service to the emergencies, it seems to be advisable to dedicate at least one room to them. With one dedicated room, an average of 1.8 emergencies would still disrupt the schedule, each day. While with two dedicated rooms, only 0.6 emergency would enter the versatile rooms. Then, to build the schedule, a central concern is to know how many operations can be planned in order to keep the overtime limited. If the manager decides that overtime is allowed once a week on average, only 13 and 11 operations can be planned, with one and two dedicated rooms, respectively. The manager wants to test if more flexibility offers a significant profit. He thus tests the case where overtime is permitted twice a week on average. In this case, three supplementary operations can be planned each day, which represents a valuable income. The manager thus decides to choose this alternative. Moreover, dedicating only one room to the emergencies leads to treatment of two supplementary elective operations each day. As the manager already measured that this configuration allows keeping the waiting time reasonnably low, he decides to organize the operating theater like this. When these decisions are taken, the manager can use the tool to get various measures. He learns that he can plan 16 operations a day, that 1.8 emergencies will disrupt the planning, leading to an occupancy rate of $95 \%$ ( $89 \%$ whitout the emergencies). Moreover, he can measure that there will be overtime twice a week, for one hour and a half on average. 
Finally, note that a similar analysis could be performed for other parameters (number of rooms, rates). For instance, if the hospital is located in a skiing region, the emergency arrival rate could increase during the winter, and could justify the decision to dedicate a second room to emergencies. The tool offers answers to such questions too.

\section{Conclusion}

In this paper, we propose an approach to help operating theater managers deal with the stochasticity of their environment. We consider the randomness brought by the operating times as well as by the arrival of emergencies. We develop an analytical approach based on a Markov process that completely models the operating theater, focusing on the behavior of the emergencies. Using simulation, we show that the assumptions required to build the Markov process have minor influence on the final analytical results. This approach allows analysis of both patient flows (elective or urgent) and to evaluate performance measures showing the quality of the service to both kinds of patients. Finally, our tool answers questions at the tactical-strategic level of decisions, such as: what is the disruption of the surgical schedule by the emergencies? What is the chance for an emergency to be treated directly? How much time will it have to wait on average? How many operations can be scheduled each working day in order to keep the overtime limited? In other words, what is the maximal occupation rate to allocate for the elective cases in the planning? What is the average overtime? What is the distribution of the working time? Is it useful to dedicate operating rooms to exclusively treat emergencies? If so, how many? What are the consequences on the quality of the service?

Concerning possible extensions of our work, although the proposed Markovian approach models the whole operating theatre, it could be appropriate to include recovery beds, which also have interesting implications. We could refine the modelling of the operations, and distinguish the operating rooms (i.e. consider different elective operating times) as well as different priority levels for the emergencies. The dispatch rule of the emergencies to the versatile rooms could be improved: the emergencies could be mainly dispatched to operating rooms treating low variance operations, for example. Our approach could also be applied to catastrophe management, where the emergencies are dispatched through a hospital network. The flow of emergencies would be transfered with priority to the nearest hospital (which corresponds to our dedicated room), but some emergencies would also be redirected to other hospitals in the network (our versatile rooms). 


\section{References}

[1] Cardoen, B., Demeulemeester, E., Belien, J., 2007. Operating room planning and scheduling: A literature review. Tech. rep., Katholieke Universiteit Leuven - Department of decision sciences and information management.

[2] Chaabane, S., 2004. Gestion prédictive des blocs opératoires. Ph.D. thesis, Institut National des Sciences Appliquées de Lyon.

[3] de Bruin, A. M., van Rossum, A. C., Visser, M. C., Koole, G. M., 2007. Modeling the emergency cardiac in-patient flow: an application of queuing theory. Health Care Management Science 10 (2), 125-137.

[4] Fackrell, M., 2007. Modelling healthcare systems with phase-type distributions. Health Care Management Science.

[5] Gerchak, Y., Gupta, D., Henig, M., 1996. Reservation planning for elective surgery under uncertain demand for emergency surgery. Management Science 42, 321-334.

[6] Gorunescu, F., McClean, S. I., Millard, P. H., 2004. Using a queueing model to help plan bed allocation in a department of geriatric medicine. Health Care Management Science 5, 307-312.

[7] Green, L., 2006. Queueing analysis in health care. In: Hall, R. (Ed.), Patient Flow: Reducing Delay in Healthcare Delivery. Springer Science, Ch. 10.

[8] Hall, R. W. (Ed.), 2006. Patient Flow: Reducing Delay in Healthcare Delivery. International Series in Operations Research \& Management Science. Springer US.

[9] Harrison, G. W., Shafer, A., Mackay, M., 2005. Modelling variability in hospital bed occupancy. Health Care Management Science, 325-334.

[10] Kao, E., Tung, G., 1981. Bed allocation in a public health care delivery system. Management Science 27, 507-520.

[11] Kim, S.-C., Horowitz, I., Young, K., Buckley, T., 1999. Analysis of capacity management of the intensive care unit in a hospital. European Journal of Operational Research 115, 36-46. 
[12] Kolker, A., 2008. Process modeling of emergency department patient flow: Effect of patient length of stay on ED diversion. Journal of Medical Systems.

[13] Komashie, A., Mousavi, A., 2005. Modeling emergency departments using discrete event simulation techniques. In: Simulation Conference, Proceedings of the Winter.

[14] Lamiri, M., Xie, X., Dolgui, A., Grimaud, F., 2008. A stochastic model for operating room planning with elective and emergency demand for surgery. European Journal of Operational Research 185, 1026-1037.

[15] Marcon, E., Kharraja, S., Simonet, G., 2003. The operating theatre planning by the follow-up of the risk of no realization. International Journal of Production Economics 85, 83-90.

[16] McClean, S., Faddy, M., Millard, P., 2006. Using markov models to assess the performance of a health and community care system. In: 19th IEEE Symposium on Computer-Based Medical Systems (CBMS'06).

[17] MEAH, 2006. Gestion et organisation des blocs opératoires dans les hôpitaux et cliniques: Recueil des bonnes pratiques organisationnelles observées. Tech. rep., Mission nationale d'Expertise et d'Audit Hospitalier, Paris.

[18] Neuts, M., 1981. Matrix-Geometric Solutions in Stochastic Models: an Algorithmic Approach. Dover Publications, New York.

[19] Preater, J., 2001. A bibliography of queues in health and medicine. Tech. rep., Mathematics Department, Keele University.

[20] Ruohonen, T., Neittaanmäki, P., Teittinen, J., 2006. Simulation model for improving the operation of the emergency department of special health care. In: Proceedings of the 38th conference on Winter simulation. California, pp. 453-458.

[21] Stewart, W., 1994. Introduction to the Numerical Solution of Markov Chains. Princeton University Press.

[22] XJ Technologies, 2007. Software AnyLogic 6. 


\section{Recent titles \\ CORE Discussion Papers}

2008/44. Geoffrey CARUSO, Dominique PEETERS, Jean CAVAILHES and Mark ROUNSEVELL. Space-time patterns of urban sprawl, a 1D cellular automata and microeconomic approach.

2008/45. Taoufik BOUEZMARNI, Jeroen V.K. ROMBOUTS and Abderrahim TAAMOUTI. Asymptotic properties of the Bernstein density copula for dependent data.

2008/46. Joe THARAKAN and Jean-Philippe TROPEANO. On the impact of labor market matching on regional disparities.

2008/47. Shin-Huei WANG and Cheng HSIAO. An easy test for two stationary long processes being uncorrelated via AR approximations.

2008/48. David DE LA CROIX. Adult longevity and economic take-off: from Malthus to Ben-Porath.

2008/49. David DE LA CROIX and Gregory PONTHIERE. On the Golden Rule of capital accumulation under endogenous longevity.

2008/50. Jean J. GABSZEWICZ and Skerdilajda ZANAJ. Successive oligopolies and decreasing returns.

2008/51. Marie-Louise LEROUX, Pierre PESTIEAU and Grégory PONTHIERE. Optimal linear taxation under endogenous longevity.

2008/52. Yuri YATSENKO, Raouf BOUCEKKINE and Natali HRITONENKO. Estimating the dynamics of R\&D-based growth models.

2008/53. Roland Iwan LUTTENS and Marie-Anne VALFORT. Voting for redistribution under desertsensitive altruism.

2008/54. Sergei PEKARSKI. Budget deficits and inflation feedback. 2008/55. Raouf BOUCEKKINE, Jacek B. KRAWCZYK and Thomas VALLEE. Towards an understanding of tradeoffs between regional wealth, tightness of a common environmental constraint and the sharing rules.

2008/56. Santanu S. DEY. A note on the split rank of intersection cuts.

2008/57. Yu. NESTEROV. Primal-dual interior-point methods with asymmetric barriers.

2008/58. Marie-Louise LEROUX, Pierre PESTIEAU and Gregory PONTHIERE. Should we subsidize longevity?

2008/59. J. Roderick McCRORIE. The role of Skorokhod space in the development of the econometric analysis of time series.

2008/60. Yu. NESTEROV. Barrier subgradient method.

2008/61. Thierry BRECHET, Johan EYCKMANS, François GERARD, Philippe MARBAIX, Henry TULKENS and Jean-Pascal VAN YPERSELE. The impact of the unilateral EU commitment on the stability of international climate agreements.

2008/62. Giorgia OGGIONI and Yves SMEERS. Average power contracts can mitigate carbon leakage.

2008/63. Jean-Sébastien TANCREZ, Philippe CHEVALIER and Pierre SEMAL. A tight bound on the throughput of queueing networks with blocking.

2008/64. Nicolas GILLIS and François GLINEUR. Nonnegative factorization and the maximum edge biclique problem.

2008/65. Geir B. ASHEIM, Claude D'ASPREMONT and Kuntal BANERJEE. Generalized timeinvariant overtaking.

2008/66. Jean-François CAULIER, Ana MAULEON and Vincent VANNETELBOSCH. Contractually stable networks.

2008/67. Jean J. GABSZEWICZ, Filomena GARCIA, Joana PAIS and Joana RESENDE. On Gale and Shapley 'College admissions and stability of marriage'.

2008/68. Axel GAUTIER and Anne YVRANDE-BILLON. Contract renewal as an incentive device. An application to the French urban public transport sector.

2008/69. Yuri YATSENKO and Natali HRITONENKO. Discrete-continuous analysis of optimal equipment replacement.

2008/70. Michel JOURNÉE, Yurii NESTEROV, Peter RICHTÁRIK and Rodolphe SEPULCHRE. Generalized power method for sparse principal component analysis.

2008/71. Toshihiro OKUBO and Pierre M. PICARD. Firms' location under taste and demand heterogeneity. 


\section{Recent titles}

\section{CORE Discussion Papers - continued}

2008/72. Iwan MEIER and Jeroen V.K. ROMBOUTS. Style rotation and performance persistence of mutual funds.

2008/73. Shin-Huei WANG and Christian M. HAFNER. Estimating autocorrelations in the presence of deterministic trends.

2008/74. Yuri YATSENKO and Natali HRITONENKO. Technological breakthroughs and asset replacement.

2008/75. Julio DÁVILA. The taxation of capital returns in overlapping generations economies without financial assets.

2008/76. Giorgia OGGIONI and Yves SMEERS. Equilibrium models for the carbon leakage problem.

2008/77. Jean-François MERTENS and Anna RUBINCHIK. Intergenerational equity and the discount rate for cost-benefit analysis.

2008/78. Claire DUJARDIN and Florence GOFFETTE-NAGOT. Does public housing occupancy increase unemployment?

2008/79. Sandra PONCET, Walter STEINGRESS and Hylke VANDENBUSSCHE. Financial constraints in China: firm-level evidence.

2008/80. Jean GABSZEWICZ, Salome GVETADZE, Didier LAUSSEL and Patrice PIERETTI. Pubic goods' attractiveness and migrations.

2008/81. Karen CRABBE and Hylke VANDENBUSSCHE. Are your firm's taxes set in Warsaw? Spatial tax competition in Europe.

2008/82. Jean-Sébastien TANCREZ, Benoît ROLAND, Jean-Philippe CORDIER and Fouad RIANE. How stochasticity and emergencies disrupt the surgical schedule.

\section{Books}

Y. POCHET and L. WOLSEY (eds.) (2006), Production planning by mixed integer programming. New York, Springer-Verlag.

P. PESTIEAU (ed.) (2006), The welfare state in the European Union: economic and social perspectives. Oxford, Oxford University Press.

H. TULKENS (ed.) (2006), Public goods, environmental externalities and fiscal competition. New York, Springer-Verlag.

V. GINSBURGH and D. THROSBY (eds.) (2006), Handbook of the economics of art and culture. Amsterdam, Elsevier.

J. GABSZEWICZ (ed.) (2006), La différenciation des produits. Paris, La découverte.

L. BAUWENS, W. POHLMEIER and D. VEREDAS (eds.) (2008), High frequency financial econometrics: recent developments. Heidelberg, Physica-Verlag.

P. VAN HENTENRYCKE and L. WOLSEY (eds.) (2007), Integration of AI and OR techniques in constraint programming for combinatorial optimization problems. Berlin, Springer.

\section{CORE Lecture Series}

C. GOURIÉROUX and A. MONFORT (1995), Simulation Based Econometric Methods.

A. RUBINSTEIN (1996), Lectures on Modeling Bounded Rationality.

J. RENEGAR (1999), A Mathematical View of Interior-Point Methods in Convex Optimization.

B.D. BERNHEIM and M.D. WHINSTON (1999), Anticompetitive Exclusion and Foreclosure Through Vertical Agreements.

D. BIENSTOCK (2001), Potential function methods for approximately solving linear programming problems: theory and practice.

R. AMIR (2002), Supermodularity and complementarity in economics.

R. WEISMANTEL (2006), Lectures on mixed nonlinear programming. 